\title{
LARYNGOLOGY
}

\section{Suture lateralisation plus arytenoid cartilage release for treating bilateral vocal fold immobility with mechanical fixation}

\author{
Lateralizzazione mediante sutura e rilascio aritenoideo per il trattamento \\ dell'immobilità cordale bilaterale con fissazione meccanica
}

\author{
W.-F. SU1, M.-C. LAN 1 , S.-C. LIU² \\ ${ }^{1}$ Department of Otolaryngology Head and Neck Surgery, Taipei Tzu Chi Hospital, Buddist Tzu Chi Medical \\ Foundation, New Taipei City, Taiwan, School of Medicine, Tzu Chi University, Hualien; ${ }^{2}$ Department of \\ Otolaryngology Head and Neck Surgery, Tri-service General Hospital, National Defense Medical Center Taipei, \\ Taiwan, Republic of China
}

\section{SUMMARY}

A suture lateralisation (SL) instead of an endolaryngeal tissue ablation procedure under endoscopy has been utilised to treat bilateral vocal fold immobility (BVFI) since 1980. However, mechanical fixation (MF) of the cricoarytenoid joint (CAJ) has continually challenged the effectiveness of glottic dilatation both in the SL procedure and the tissue ablation procedure. From 2007 to 2015 , a total of 38 patients with BVFI underwent 40 exo-endolaryngeal suture lateralisation (exoeSL) procedures and three were diagnosed with MF in our hospital. For these MF, we introduced an external approach method to release the CAJ followed by a similar exoeSL procedure. The CAJ release procedure enabled the preservation of the endolaryngeal mucous membrane (ELM) and consequently spared the use of laser surgery. All three CAJ release procedures led to decannulations (one patient) or improvement of dyspnoea (two patients). The difference between the exoeSL and the endo-exolaryngeal suture lateralisation (endoeSL) procedure is discussed based on their effectiveness in MF management.

KEY WORDS: Suture lateralisation • Bilateral vocal fold immobility • Cricoarytenoid joint fixation • Arytenoidectomy • Arytenoid cartilage release

\section{RIASSUNTO}

La lateralizzazione mediante sutura al posto dell'ablazione di tessuto endolaringeo per via endoscopica è una procedura utilizzata per trattare la paralisi cordale bilaterale fin dal 1980. Tuttavia, la fissazione meccanica dell'articolazione cricoaritenoidea ha continuamente contrastato l'efficacia della dilatazione glottica, sia nella procedura di lateralizzazione mediante sutura che nell' exeresi di tessuto endolaringeo. Dal 2007 al 2015, un totale di 38 pazienti con paralisi cordale bilaterale sono stati sottoposti a 40 lateralizzazioni mediante sutura eso-endolaringea (exoeSL) e 3 di questi pazienti hanno avuto diagnosi di fissità meccanica nel nostro ospedale. Abbiamo introdotto un approccio esterno per rilasciare la giunzione cricoaritenoidea seguito da una procedura exoeSL simile. Questa procedura di rilascio della giunzione cricoaritenoidea ha permesso la preservazione della membrana mucosa endolaringea, risparmiando quindi l'utilizzo della chirurgia laser. Tutte e tre le procedure di rilascio della giunzione cricoaritenoidea hanno portato a decannulazione (1 paziente) o a un miglioramento della dispnea (2 pazienti). La differenza tra la lateralizzazione exoeSL e endo esolaringea saranno quindi discusse in base all' efficacia nel trattamento della fissità meccanica.

PAROLE CHIAVE: Sutura di lateralizzazione $\bullet$ Paralisi cordale bilaterale $\bullet$ Fissità dell' articolazione cricoaritenoidea $\bullet$ Aritenoidectomia, rilascio cartilagine aritenoidea

\section{Introduction}

A number of so-called minimally invasive operations carried out with endoscopy have been proposed for treating BVFI ${ }^{1-4}$. They have been generally categorised into two groups. The first is an irreversible endolaryngeal tissue ablation procedure ${ }^{12}$ using a laser beam. The second is an ELM preservation procedure using an SL procedure with specially designed instruments to achieve endoeSL ${ }^{23}$ or without it to perform an exoeSL procedure ${ }^{4-8}$.

BVFI is a term used to describe vocal cords that are restricted secondary to neuropathy, muscular disorders, or MF. 
MF has continually challenged more or less the all aforementioned interventions on their principles. The tension on the vocal fold from the MF seems to work against the effectiveness of the SL procedure. As for this, in the setting of the endoeSL procedure, while Rovo et $\mathrm{al}^{3}$ released the MF using an endolaryngeal scythe, Lichenberger et $\mathrm{al}^{9}$ and Castellanos et al. ${ }^{10}$ treated it using laser arytenoidectomy. In the setting of the exoeSL procedure, we have also introduced an external approach method to release the MF and retain the integrity of the ELM. With this major refinement of surgical technique in the cricoarytenoid joint (CAJ) mobility, the indications of exoeSL can be extended from neurological vocal immobility to MF. Moreover, exoeSL and endoeSL procedure will be discussed in terms of their effectiveness in MF management.

\section{Materials and methods}

From 2007 to 2015, a total of 38 patients with BVFI due to various causes underwent 40 exoeSL procedures, with five being diagnosed with MF, two patients postoperatively and three preoperatively. Consequently, a total of three MFs (two females and one male aged from 52 to 66 with mean 57) with two MFs due to previous laser surgeries and one MF due to intubations were treated with CAJ release and the SL procedure.

\section{Evaluation of respiratory function}

To objectively evaluate the improvement of respiratory function, pulmonary function test with flow-volume loop was performed before and 2 months after the operation. The ratio of maximal mid-expiratory flow to maximal mid-inspiratory flow, termed the mid-vital capacity flow ratio, was used to represent the severity of BVFI-related variable extrathoracic airway obstruction ${ }^{11}$.

\section{Evaluation of phonatory performance}

An early and a late postoperative voice assessment were performed 2 and 9 months, respectively, after the operation using a perceptual rating scale for voice quality in two patients. The voice quality in terms of loudness, breathness and hoarseness was rated as (-); no change, (+); mildly, $(++)$; moderately and $(+++)$; severely deteriorated voice.

\section{Evaluation of swallowing function}

Swallowing function was evaluated subjectively. Subjects with clinical aspiration or history of cerebral stroke were excluded from this surgery, regardless of a preexisting tracheostomy tube or not. Swallowing function was simply rated as tolerable or intolerable aspiration based on clinic symptoms.

\section{Technique description}

The surgeons' real know-how lies in the method of achieving vocal fold lateralisation without any endolaryngeal soft tissue and the associated ELM loss. The first step is an open surgery for arytenoid cartilage release, which proceeds as follows. (1) A horizontal incision is made at the level of the lower edge of the thyroid cartilage, extending $5 \mathrm{~cm}$ laterally from the midline on the lateralisation side and $0.5 \mathrm{~cm}$ on the other side. (2) Strap muscles and the inferior constrictor muscle are divided to expose the oblique line and posterior edge of the thyroid lamina. (3) The inferior constrictor muscle and its inner lining; pyriform sinus mucosa (Fig. 1 grey line) are dissected from the thyroid cartilage (Fig. 1) in a lateral-to-posteromedial direction until the muscular process can be palpated. (4) The CAJ is identified and the arytenoid cartilage (arrow in Fig. 2) is completely separated from the cricoid cartilage (arrow head in Fig. 2). (5) The depth of the paraglottic wound space (Fig. 1 grey dotted line) is then measured from the posterior edge of the thyroid lamina to the deepest end of the space (Fig. 1 "B"). (6) A mark as a reference for the injection needle entrance is then made on the thyroid lamina, which should be at least

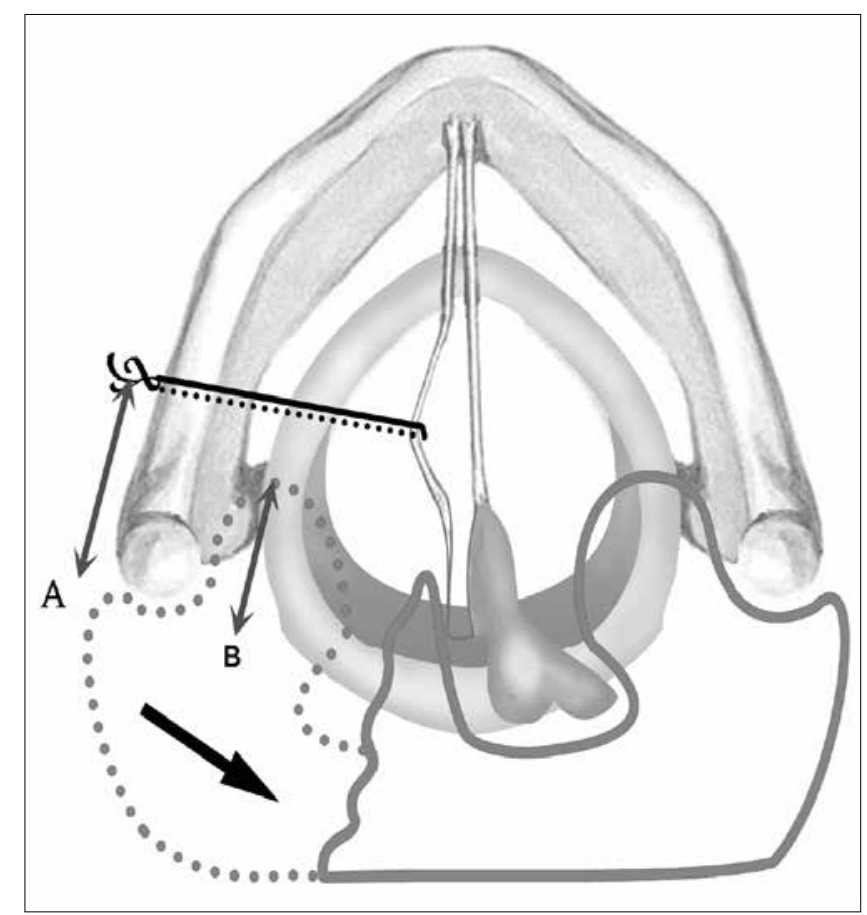

Fig. 1. The scheme illustrates the surgical method with arytenoid cartilage release through the paraglottic space plus suture lateralisation procedure. The surgical wound in the paraglottic space (grey dotted line) was created after the pyriform sinus mucosa (grey line) had been peeled off from the thyroid lamina laterally and the paraglottic space medially. Note the suture canal is outside the wound space. A: the distance from the entrance of the injection needle on the thyroid lamina to the posterior margin of the thyroid lamina. $B$ : the distance from the deepest wound bed in the paraglottic space to the posterior margin of the thyroid lamina. 


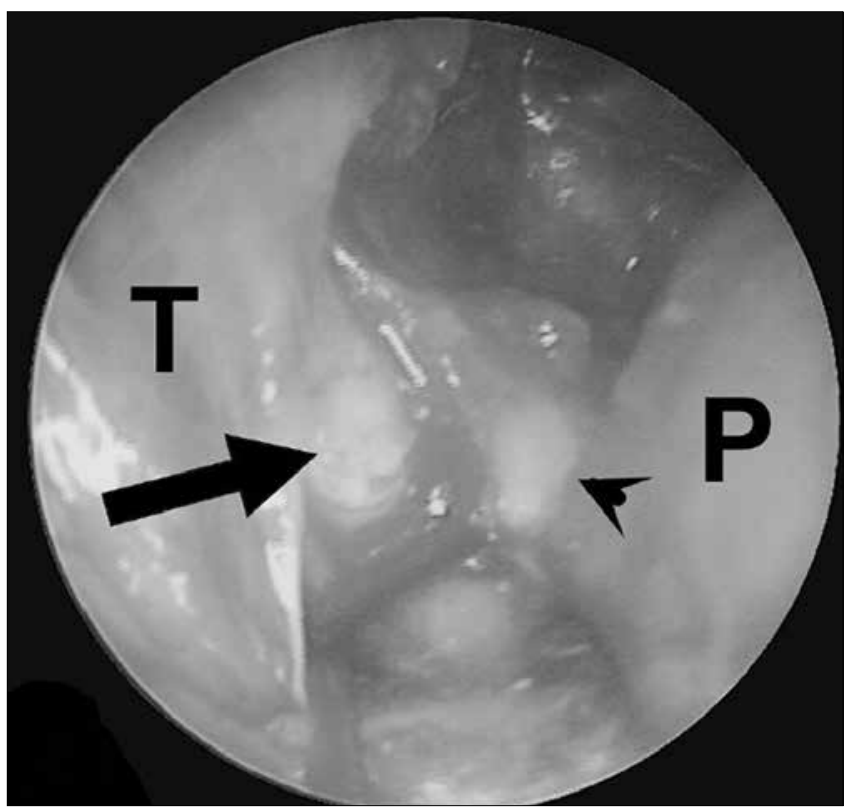

Fig. 2. The CAJ cavity is opened. The black arrow indicates the arytenoid cartilage and the arrow head indicates the cricoid cartilage. P: the pyriform sinus mucosa. T: the thyroid lamina.

$2 \mathrm{~mm}$ anterior to the deepest end of the space and measured as " $\mathrm{L}$ " in the Fig. 1. This needle entrance and its stitch canal in the paraglottic soft tissue will be outside the wound space, avoiding the infection from the glottic lumen.

The second step is an exoeSL procedure after the larynx is exposed microscopically using a $0^{\circ}$ telescopy. This was well described in our previous publication ${ }^{4}$. This study has been approved by the institutional review board.

\section{Results}

\section{Respiration}

All three arytenoid release procedures obtained decannulations (one patient) (Fig. 3) or improved dyspnoea (two patients). The mean mid-vital capacity flow ratio was 3.54 and 4.33 before the operation and was reduced to 0.89 and 1.33 , respectively, after the operations in two completed flow-volume loop examinations.

\section{Phonation}

In the early postoperative voice assessment, two non-tracheostomised patients obtained moderately deteriorated voice $(++)$ because of large glottal gaps. In the late postoperative voice assessment, two patients obtained mildly changed voice quality $(+)$ because of ventricular compensation.

\section{Evaluation of swallowing function}

All three subjects had temporally mild or even no aspira- tion for a few days after the operation, but it disappeared spontaneously. The mean follow-up time was 17.1 months (range 13 to 21 months).

\section{Discussion}

The external approach to the CAJ was proposed to medialise the vocal fold in 1978 by Ishiki et al. ${ }^{12}$. On the contrary, Woodman et al. ${ }^{13}$ lateralised the vocal fold through the same approach in 1946, which was modified by Shetty et al. ${ }^{14}$ in 1998. They sutured on the vocal ligament and the thyroarytenoid muscle through the paraglottic space and fixed them posteriorly on the inferior horn or horizontally to the posterior edge of the thyroid lamina, respectively. The suture on the vocal ligament through the narrow paraglottic space appeared to be difficult. We performed the exoeSL through the thyroid lamina instead of the narrow paraglottic space to simplify the surgical access to the vocal fold if the CAJ is mobile, as in previous publications ${ }^{2-8}$. However, if the CAJ is fixed, this makes the endoscopic vocal fold lateralisation procedures more challenging. Thus, we released the arytenoid cartilage from the CAJ using the external approach at first and then the exoeSL procedure was used. Compared with the other exoeSL or endoeSL procedures, this arytenoid release procedure can keep the ELM intact and spare the use of laser surgery. All three MFs had a completely mobile CAJ from this circumferential arytenoid release procedure and subsequent decannulations (one patient) or improvement in dyspnoea (two patients). Compared to the endoscopic arytenoid lateropexy proposed by Rovo et al. ${ }^{3}$, the endolaryngeal scythe could only have separated the anterior aspect of the $\mathrm{CAJ}$, which was unable to completely resolve the MF aris-

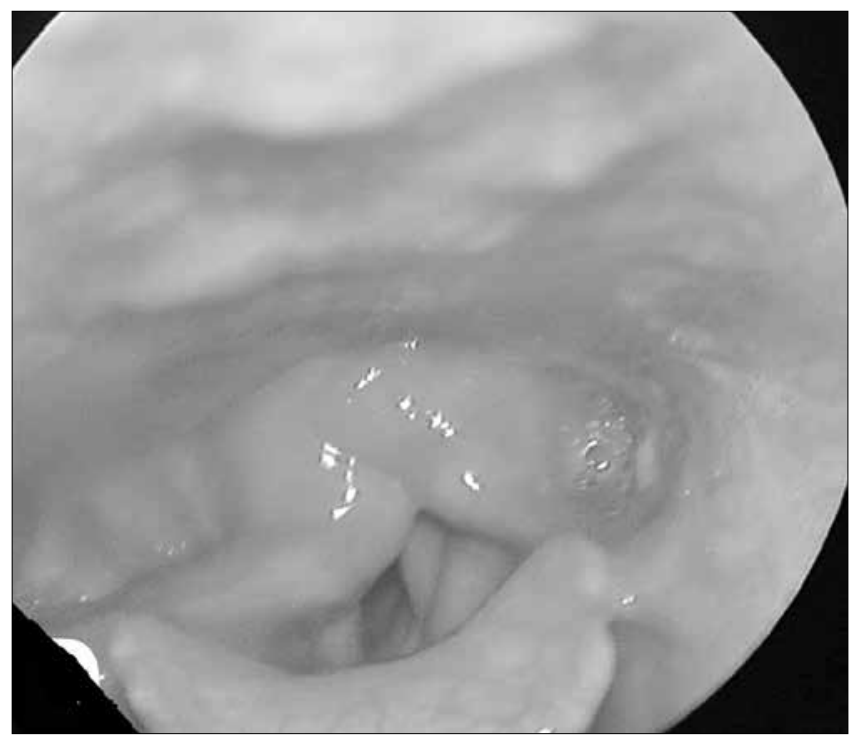

Fig. 3. Endoscopy shows the glottic lumen one year after the operation. 
ing from the rheumatoid arthritis or irradiation ${ }^{3}$. In patients with grade I or II isolated posterior glottal stenosis (PGS), the endoscopic arytenoid lateropexy might be highly recommended because of the expectation of future vocal mobility. However, in patients with PGS grade III or IV, surgical release itself may further produce joint capsule scarring. Not only will the future vocal movement be unpredictable, but also the mobility of the CAJ enhanced by the scythe will be queried. Similarly, the postcricoid mucosal advancement flap proposed by Castellanos et al. ${ }^{10}$ was only appropriate in grade II PGS because the laser arytenoidectomy they used to treat the grade III and IV PGS was still unable to maintain the integrity of the ELM, as proposed by Litchenberger et al. 29 . In addition, revisions were usually required for granulation tissue formation after laser surgeries ${ }^{315}$. The disadvantages of this arytenoid release procedure include insufficient ventricular compensation for the voice due to the excessive glottic enlargement, compared with the simple SL procedure without arytenoid release ${ }^{4}$. Compared to the tissue ablation procedure (laser arytenoidectomy), the functional outcomes including the respiratory, phonatory and swallowing functions varied with the magnitude of tissue ablation and tissue regrowth. On the other hand, an intact ELM coupled with a tension-free suture loop might warrant the consistent effectiveness in the respiratory function. However, ventricular compensation longer than 9 months might be required for the breathing voice. As for swallowing function, the coordination of tongue-pharyngolaryngeal complex still play a major role in the aspiration after operation, especially in elderly patients.

\section{Conclusions}

With this major refinement of surgical technique in CAJ mobility, not only can the exoeSL procedure extend its indications to MF, but it also poses a better chance of avoiding granulation tissue formation due to laser surgery. Therefore, exoeSL with arytenoid cartilage release appears to be a viable or even essential option in some patients with severe MFs and merits further investigation and advocacy in the future. Nevertheless, patient selection should depend on various factors, including patient's degree of upper airway obstruction, voice demands and quality of life priorities.

\section{References}

1 Dennis DP, Kashima H. Carbon dioxide laser posterior cordectomy for treatment of bilateral vocal cord paralysis. Ann Otol Rhinol Laryngol 1989;98:930-4.

2 Lichtenberger G. Reversible lateralization of the paralyzed vocal cord without tracheostomy. Ann Otol Rhinol Laryngol 2002;111:21-6.

3 RoVo L, Madani S, Sztanò B, et al. A new thread guide instrument for endoscopic arytenoid lateropexy. Laryngoscope 2010;120:2002-7.

$4 \mathrm{Su} \mathrm{WF}$, Liu SC. Suture lateralization in patients with bilateral vocal cord paralysis. J Voice 2014;28:644-51.

5 Songu M, Aslan H, Denizoglu I, et al. Vocal and ventricular fold lateralization using crossing sutures with the thyroplasty window technique for bilateral vocal fold immobility: longterm results. Acta Otolaryngol 2013;133:1201-6

6 Ejnell H, Mansson I, Hallen O, et al. A simple operation for bilateral vocal cord paralysis. Laryngoscope 1984;94:954-8.

7 Katilmis H, Ozturkcan S, Basoglu S, et al. New technique for the treatment of bilateral vocal cord paralysis: vocal and ventricular fold lateralization using crossing sutures with thyroplasty technique. Acta Otolaryngol 2011;131:303-9.

8 Ezzat WF, Shehata M, Kamal I. Adjustable laterofixation of the vocal fold in bilateral vocal fold paralysis. Laryngoscope 2010;120:731-3.

9 Lichtenberger G. Comparison of endoscopic glottis-dilating operations. Eur Arch Otorhinolaryngol 2003;260:57-61.

10 Castellanos PF, Atallah I, Krishnah M, et al. Reconstructive transoral laser microsurgery for posterior glottal web with stenosis. Laryngoscope 2017;127:685-90.

11 Kirchner FR. Endoscopic lateralization of the vocal cords in abductor paralysis of the larynx. Laryngoscope 1979;89:1779-83.

12 Isshiki N, Tanabe M, Sawada M. Arytenoid adduction for unilateral vocal cord paralysis. Arch Otolaryngol 1978;104:555-8.

13 Woodman, D. A modification of the extralaryngeaI approach to arytenoidectomy for bilateral vocal cord palsy. Arch Otolaryngol 1946;43:63-5.

14 Shetty SC, Prasad D. Woodman's operation for bilateral vocal cords paralysis- Our experience. Indian J Otolaryngol Head Neck Surg. 1998;50:241-5.

15 Bottero S, Peradotto F, Roma R, et al. Laryngotracheal reconstruction in glottic-subglottic stenosis associated with DiGeorge syndrome in a four and a half-month-old infant. Acta Otorhinolaryngol Ital 2015;35:53-7. 